\title{
IL-1及 inhibition in autoimmune inner ear disease: can you hear me now?
}

\author{
Steven D. Rauch ${ }^{1,2}$ \\ 'Department of Otology and Laryngology, Harvard Medical School, Boston, Massachusetts, USA. ${ }^{2}$ Vestibular Division, Otolaryngology Department, Massachusetts Eye and Ear Infirmary and \\ Massachusetts General Hospital, Boston, Massachusetts, USA.
}

\begin{abstract}
Clinical vignette: A 51-year-old man with right-sided sudden hearing loss presents to the otology clinic. He has a 4-year history of episodic vertigo of several hours' duration and fluctuating, progressive sensorineural hearing loss in his left ear. The vertigo attacks have not occurred for the last 18 months, and the left ear hearing is consistently poor. The patient's right ear hearing has dropped in the last 36 hours. MRI imaging of brain and temporal bone are normal. A 2-week "burst and taper" of oral prednisone is administered with no effect. Over the next 3 months, serial audiograms show rapidly progressive loss of threshold and word recognition scores on the right side. A trial of high-dose prednisone ( $60 \mathrm{mg} / \mathrm{d}$ for 30 days) results in full recovery of the right ear hearing and substantial improvement in the left ear. As the prednisone dose is slowly tapered over several months, the hearing drops again.
\end{abstract}

The patient above is likely suffering from autoimmune inner ear disease (AIED), a rare form of sensorineural hearing loss (SNHL). In 1979, McCabe provided the first clinical evidence that SNHL could have an autoimmune component (1), supporting the hypothesis put forth by Lehnhardt (2). Despite multiple confirmatory reports that have refined the clinical characterization of AIED, the pathogenesis of this very uncommon condition remains vague and controversial, in large part because there are no universally accepted diagnostic criteria or tests. A loose consensus has gradually arisen that AIED presents as an idiopathic, rapidly progressive, bilateral SNHL. AIED patients exhibit a loss of hearing that occurs over a period of weeks to months, too slow to be considered "sudden" SNHL and too fast to be classified as age-related or other genetically determined degenerative SNHL. Serial audiometry dem- onstrates progression, which is usually monotonic but may fluctuate. Typically the ears progress asynchronously, often years apart. In many cases, deafness in the first ear may be congenital or the result of some identifiable cause prior to rapid hearing loss in the second ear, raising the possibility of AIED. This rapidly progressive asynchronous hearing loss alone is considered sufficient by some to make an AIED diagnosis, whereas others require evidence of steroid responsiveness.

\section{Current therapies}

A classification scheme put forth by Harris et al. defines type 1 AIED as an idiopathic, rapidly progressive, bilateral SNHL with no evidence of other systemic autoimmune or inflammatory disease (Table 1 and ref. 3). A recent retrospective of 47 AIED cases by Matsuoka and Harris provides an excellent summary of the clinical epidemiology (4). In contrast to many sys-

\section{Related Article: p. 4115}

Conflict of interest: Steven D. Rauch receives consulting fees from BestDoctors, Inc. His spouse receives consulting fees from Blend Therapeutics and Eleven Biotherapeutics and holds stock in Biogen Idec. Reference information: J Clin Invest. 2014;124(9):3685-3687. doi:10.1172/JCI77197.

temic autoimmune diseases, type 1 AIED equally affects males and females. Type 1 AIED onset typically occurs in the mid50 s and seems to be far more common in white (non-Hispanic) populations than in other racial/ethnic groups. Moreover, all type 1 AIED patients in the Matsuoka and Harris cohort exhibited aural pressure and tinnitus. Approximately 15\% experienced vestibular symptoms of vertigo, disequilibrium, or dizziness.

Audiometric diagnosis of AIED typically consists of an auditory pure tone threshold shift of at least $15 \mathrm{~dB}$ at 1 test frequency or $10 \mathrm{~dB}$ at 2 or more consecutive test frequencies, or a greater than $12 \%$ drop in word recognition within a 3-month period. Often a single month-to-month comparison does not meet these criteria, but comparison of monthly audiograms over a 3-month span will clearly demonstrate progressive hearing loss. Unlike idiopathic sudden SNHL, which is an otologic emergency and has only a 2- to 4 -week window for successful corticosteroid treatment (5), AIED appears to be steroid responsive for weeks to months. Unfortunately, once AIED hearing loss stabilizes, corticosteroids appear to no longer be effective (S.D. Rauch, unpublished observations).

Currently, the cornerstone of AIED therapy is corticosteroids. Typically, patients are given a therapeutic trial of oral prednisone, which is administered for 30 days, and hearing is reassessed. If there is a steroid response, patients are slowly tapered off of the corticosteroid. Each month, hearing is tested to confirm stability or improvement before further lowering the dose. Once the dose reaches $10 \mathrm{mg} / \mathrm{d}$, this dose is continued for 3 months before the final taper to discontinue the medication; therefore, treatment duration is 6-12 months. If a patient exhibits hearing loss during any month of treatment, the current dose may be held for an additional month to see if the hearing will rise again. Alternatively, the dose can be bumped 


\section{Table 1. Harris AIED classification}

Type

1: Organ-specific (ear)

\section{(1)}

\section{2: Rapidly progressive bilateral SNHL with systemic autoimmune disease}

\section{3: Immune-mediated Meniere's disease}

5: Immune-mediated SNHL with other discrete organ system disease, such as Cogan's syndrome, Wegener's granulomatosis, and relapsing polychondritis

6: Nonimmune rapidly progressive SNHL (ototoxic or paraneoplastic syndrome)
4: Rapidly progressive bilateral SNHL with associated inflammatory disease, such as chronic otitis media, Lyme disease, and otosyphilis

\section{Features}

Rapidly progressive bilateral SNHL

All ages, although middle age most common

No other clinical evidence of autoimmune disease

Negative serology as evaluated by antinuclear antibody, erythrocyte sedimentation rate, rheumatoid

factor, and $\mathrm{C} 1 \mathrm{q}$ binding assay

$>50 \%$ of patients respond to high-dose corticosteroids

\section{Rapidly progressive bilateral SNHL}

Other autoimmune condition present, such as systemic lupus erythematosus, ulcerataive colitis,

polyarteritis nodosa, vasculitis, rheumatoid arthritis, and Sjögren's disease

Hearing loss often worse with flare-up of autoimmune condition

Serologic studies positive in accordance with systemic autoimmune disease

Steroid responsive, might be managed with targeted therapies for underlying illness

Bilateral fluctuating SNHL with predominant episodic vestibular symptoms

Subset of patients with contralateral delayed endolymphatic hydrops or recent instability of better

hearing ear in a patient with burned out Meniere's disease

Steroid responsive, might require long-term immunosuppression due to relapses

\section{Evidence of profound drop in hearing with longstanding chronic otitis media} Might show inflammation and perforations of tympanic membrane Hearing loss progresses despite treatment of the infectious agent

Serological tests might be positive for underlying disease, patients should be evaluated for granulomatous disease and vasculitis by biopsy of tissue available

Steroid responsive, might require long-term immunosuppression

Serologic tests may be positive in accordance with associated disease Initially responsive to high-dose steroid, but tends to become resistant over time and with each relapse

Young to elderly patients with idiopathic rapidly progressive SNHL leading to deafness

Severe ear pain, pressure, tinnitus, and some imbalance

Negative serology

Inciting event common; drug-related (e.g., oxycodone), paraneoplastic syndrome

No response to corticosteroids or antiviral treatment

As described by Harris et al. (3).

back up until hearing stabilizes. Treatment with a total duration of less than 6 months is associated with a $100 \%$ relapse rate.

There are 3 possible outcomes for steroid-responsive AIED patients, the least likely of which is a successful taper with no further hearing problems. Most commonly, patients are successfully tapered off corticosteroids, but relapse or exhibit gradual, progressive hearing loss over the years. Some patients will have an outcome that falls between these extremes. These patients are considered to be steroid dependent, meaning that their hearing is stable while on steroids, but declines whenever the dose gets too low. In this patient subset, there is considerable intersubject variability in the threshold prednisone dose required to maintain hearing stability. In steroid-dependent cases, it is often possible to introduce another immunomodulatory drug like a TNF- $\alpha$ inhibitor, such as etanercept, infliximab, or adalimumab, and then successfully withdraw the corticosteroid. In some cases, the TNF- $\alpha$ inhibitor can be successfully withdrawn. Older and better-tolerated steroid-sparing agents, like methotrexate, have been evaluated for this purpose, but have been unsuccessful (6). For patients unresponsive to the initial 30-day prednisone trial, no other effective treatments are available.

\section{Knowledge gap}

It is not clear exactly how the immune system damages the inner ear. It has long been thought that the inner ear is an immunologically privileged site protected from systemic immune system by a "bloodlabyrinth barrier," akin to brain and bloodbrain barrier; however, this notion has been disproven. Animal models have shown that systematic immunization with inner ear antigens produces inner ear dysfunc- tion $(7,8)$. Furthermore, a circulating antibody was identified in AIED patients that is immunoreactive with a $68-\mathrm{kDa}$ inner ear antigen (9), which is likely HSP70 (10, 11). Efforts to assay anti-HSP70 antibodies as a clinical diagnosis and monitoring tool for AIED were initially hopeful; however, more recent analysis revealed that the sensitivity and specificity of this test is too low to be of clinical value (4). Multiple groups have identified circulating antibodies that target an inner ear protein found in supporting cells of the organ of Corti within the cochlea $(12,13)$ in $30 \%-40 \%$ of AIED patients. The antigen was eventually identified as the transmembrane protein choline transporter-like protein 2 (CTL2). A clinical correlation between the presence of anti-CTL2 antibodies and steroid responsiveness appeared robust in a small cohort of subjects, but no assay has been made available for clinical use. 


\section{Research advance}

Previously, Vambutas and colleagues collected inner ear perilymph samples during cochlear implantation in patients with profound deafness from end-stage AIED and used this perilymph to stimulate autologous peripheral blood monocytes (PBMCs). Compared with perilymph from control cochlear implant patients, perilymph from individuals with AIED induced the expression of interleukin-1 type II decoy receptor (IL-1R2) in PBMCs (14). Subsequent studies of PBMCs revealed increased IL-1 $\beta$ expression in steroid-resistant versus steroidresponsive AIED patients. Furthermore, the glucocorticoid dexamethasone was unable to prevent IL-1 $\beta$ release, but the IL-1 receptor antagonist anakinra effectively did (15). These observations shed new light on possible mechanisms of immunologic damage in AIED as well as offer a potential novel treatment with anakinra or other IL-1 $\beta$ inhibitors or receptor antagonists.

In this issue of the JCI, Vambutas et al. present results of an open-label, singlearm, phase I/II pilot study of anakinra for AIED patients that are unresponsive to corticosteroids (16). Of patients that completed an 84-day treatment, 7 of 10 showed at least a transient hearing improvement while receiving anakinra. Some of the subjects, deemed treatment responders, went on to exhibit a drop in hearing despite continuation of treatment. In each of these fluctuating cases, plasma IL-1 $\beta$ levels vacillated up and down in concordance with hearing. While the study by Vambutas and colleagues only evaluated a very small cohort, and numerous details in the methodology are debatable, it provides a potential treatment with demonstrated efficacy in corticosteroid nonresponders. Moreover, the correlation of hearing levels and plasma IL-1 $\beta$ levels corroborates earlier work that supports a role for cytokines in the etiopathogenesis of AIED.

\section{Recommendations}

While AIED is a very rare condition, it represents one of the few examples of potentially reversible SNHL. For that reason, and because of the insights that may be gained from pursuing treatment of AIED and other SNHLs with cytokine modulators, the present report by Vambutas et al. (16) is exciting. Additionally, this report indicates that IL-1 $\beta$ has potential as a clinical marker to evaluate treatment response and predict outcome. Further prospective, randomized, multicenter clinical trials are now needed to confirm and extend these preliminary results.

Address correspondence to: Steven D. Rauch, Massachusetts Eye and Ear Infirmary, 243 Charles Street, Boston, Massachusetts 02114, USA. Phone: 617.573.3644; E-mail: steven_rauch@meei.harvard.edu.

1. McCabe BF. Autoimmune sensorineural hearing loss. Ann Otol Rhinol Laryngol. 1979; 88(5 pt 1):585-589.

2. Lehnhardt von E. Plötzliche Hörstörungen auf beiden Seiten Gleichzeitig oder nacheinander aufgetreten. Z Laryngol Rhinol Otol. 1958;37(1):1-16.

3. Harris JP, Keithley EM, Gopen Q. Autoimmune inner ear disease and other autoimmune diseases with inner ear involvement. In: Snow JB, Ballenger JJ, eds. Otolaryngology Head and Neck Surgery. Hamilton, Ontario, Canada: Decker Intellectual Properties; 2002:396-407.
4. Matsuoka AJ, Harris JP. Autoimmune inner ear disease: a retrospective review of forty-seven patients. Audiol Neurotol. 2013;18(4):228-239.

5 . Rauch SD. Idiopathic sudden sensorineural hearing loss. N Engl J Med. 2008;359(8):833-840.

6. Harris JP, et al. Treatment of corticosteroidoresponsive autoimmune inner ear disease with methotrexate. JAMA. 2003;290(14):1875-1883.

7. Beikert P. Zur Frage der EmpfindungsSchwerhörigkeit und Autoallergie. Z Laryngol Rhinol Otol. 1961;40:837-842.

8. Terayama Y, Sasaki Y. Studies on experimental allergic (isoimmune) labyrinthitis in guinea pigs. Acta Otolaryngol. 1964;58:49-64.

9. Harris JP, Sharp PA. Inner ear autoantibodies in patients with rapidly progressive sensorineural hearing loss. Laryngoscope. 1990;100(5):516-524.

10. Billings PB, Keithley EM, Harris JP. Evidence linking the 68 kilodalton antigen identified in progressive snesorienural hearing loss patient sera with heat shock protein 70. Ann Otol Rhinol Laryngol. 1995;104(3):181-188.

11. Bloch DB, San Martin JE, Rauch SD, Mosckcki RA, Bloch KJ. Serum antibodies to heat shock protein 70 in sensorineural hearing loss. Arch Otolaryngol Head Neck Surg. 1995;121(10):1167-1171.

12. Nair TS, et al. Identification and characterization of choline transporter-like protein 2, an inner ear glycoproteins of 68 and $72 \mathrm{kDa}$ that is the target of antibody-induced hearing loss. J Neurosci. 2004;24(7):1772-1779.

13. Kommareddi PK, et al. Autoantibodies to recombinant human CTL2 in autoimmune hearing loss. Laryngoscope. 2009;119(5):924-932.

14. Vambutas A, DeVoti J, Goldofsky E, Gordon M, Lesser M, Bonagura V. Alternate splicing of interleukin-1 receptro type II (IL1R2) in vitro correlates with clinical glucocorticoid responsiveness in patients with AIED. PLoS One. 2009;4(4):e5293.

15. Pathak S, Goldofsky E, Vivas EX, Bonagura VR, Vambutas A. IL-1 $\beta$ is overexpressed and aberrantly regulated in corticosteroid nonresponders with autoimmune inner ear disease. J Immunol. 2011;186(3):1870-1879.

16. Vambutas A, et al. Early efficacy trial of anakinra in corticosteroid-resistant autoimmune inner ear disease. J Clin Invest. 2014;124(9):4115-4122. 